\title{
Rectifying "nanohomo" contacts of W-Ga-C composite pad and nanowire fabricated by focused-ion-beam-induced chemical vapor deposition
}

\author{
Wuxia Li and Tiehan H. Shen ${ }^{\text {a) }}$ \\ Joule Physics Laboratory, Institute for Materials Research, University of Salford, Salford, \\ M5 4WT, United Kingdom
}

(Received 15 April 2005; accepted 27 July 2005; published online 16 September 2005)

\begin{abstract}
We prepared $\mathrm{W}-\mathrm{Ga}-\mathrm{C}$ composite contacts on $\mathrm{W}-\mathrm{Ga}-\mathrm{C}$ composite nanowires by focused-ion-beam-induced chemical vapor deposition using a dual-beam scanning electron microscope/focused-ion-beam system. The current-voltage $(I-V)$ characteristics of wires were found to change from nonlinear to linear with increasing wire thickness. For wires with small dimensions, which result in strong nonlinear $I-V$ behavior at room temperature, pairs of contacts were fabricated along the wire under different ion energies and scanning modes. Nonlinear and asymmetric rectifying $I-V$ characteristics were observed. The results suggest that nanoscaled $\mathrm{W}-\mathrm{Ga}-\mathrm{C}$ nanowires may behave similarly to semiconductors and that the contact characteristics may be modified using different deposition conditions. Furthermore, ohmiclike junctions could be formed through the use of specific deposition conditions for the contact pads and nanowires. (C) 2005 American Institute of Physics. [DOI: 10.1063/1.2051798]
\end{abstract}

In recent years, nanowires and tubes have attracted much attention, as they serve not only as a base for studying lowdimensional phenomena, but also as building blocks for nanodevices and circuits. ${ }^{1-3}$ Although carbon nanotubes have been the most extensively studied, ${ }^{4-6}$ work has also been reported on semiconductor nanowires such as $\mathrm{ZnO}$ (Ref. 7), GaN (Ref. 8), and InP (Ref. 1). However, little exists in the literature on the applications of metallic nanowires as building blocks for nanodevices. ${ }^{9}$ The use of a focused beam of either electron or ion to induce the deposition of materials from metalorganic precursors has recently emerged as an important technique to fabricate nanowires and nanowire-based devices. $^{9-12}$ The method makes it possible to form interconnecting complex functional units in a single "writing" step, with a resolution comparable to the structures defined by the electron-beam lithography. So far, most of such studies have been focused on the fabrication, ${ }^{13,14}$ the electrical resistivity, ${ }^{15,16}$ and the current-voltage $(I-V)$ characteristics of these nanowires. ${ }^{17}$

In the present paper, we report on a study of $\mathrm{W}-\mathrm{Ga}-\mathrm{C}$ composite contacts to $\mathrm{W}-\mathrm{Ga}-\mathrm{C}$ nanowires, which we shall refer to as "nanohomo" contacts, fabricated by Ga ion-beaminduced chemical vapor deposition (CVD) using a single metalorganic precursor. The $I-V$ characteristics revealed that the contact properties of the $\mathrm{W}-\mathrm{Ga}-\mathrm{C}$ pads to the $\mathrm{W}-\mathrm{Ga}-\mathrm{C}$ nanowires were sensitive to deposition parameters and that rectifying contact pairs were achievable, indicating that the use of focused ion- and/or electron-beam-induced CVD is a prominent method for the fabrication of metallic or hybrid metal-nanowire-based devices and circuits.

A dual-beam scanning electron microscope (SEM)/ focused-ion-beam (FIB) system supplied by JEOL was used for the fabrication. It comprises an FIB unit (Orsay Physics "Canion 31+column"), an Orsay Physics gas injection module, an SEM (JSM-5910 with $\mathrm{LaB}_{6}$ filament), and an additional RAITH lithographic software package. The electrical

\footnotetext{
${ }^{\text {a) }}$ Author to whom correspondence should be addressed; electronic mail:
} t.shen@salford.ac.uk properties were measured at room temperature with a Keithley 230 programmable voltage source and a 6517 electrometer.

Si wafers were used as the substrate for fabricating the devices. Prior to the FIB processing, a $67-\mathrm{nm}$-thick $\mathrm{Si}_{3} \mathrm{~N}_{4}$ insulating layer was deposited by CVD over the entire $p$-type Si substrate. To avoid damaging the substrate surface by the $\mathrm{Ga}$ ion beam, both the electron and ion beams were focused and aligned on neighboring features away from the fabrication areas. Extra care was taken in the subsequent motion of the sample stage so that the alignment was maintained and the desired patterns could be deposited in an area of the substrate that was otherwise unaffected by the Ga ion beam.

The fabrication details are as follows: (a) Large 40 $\times 40 \mu \mathrm{m}^{2}$ contact pads were fabricated with an estimated dose of $9.1 \times 10^{17}$ ions $/ \mathrm{cm}^{2}$ at $30 \mathrm{keV}$ using the ORSAY PHYSICS software. (b) Wide interconnecting strips (width: $0.63 \mu \mathrm{m})$ with different thicknesses were made at lower ion energy (with an accelerating voltage of $20 \mathrm{keV}$ and a probe current of $39 \mathrm{pA}$ ). Different thicknesses were achieved using different numbers of beam scanning loops (20,40, and 60) with corresponding estimated doses of $5.8 \times 10^{18}, 1.2 \times 10^{19}$, and $1.7 \times 10^{19}$ ions $/ \mathrm{cm}^{2}$, respectively. (c) More complex structures, as shown in Fig. 1, with different types of interconnecting wires to create asymmetric contact pairs were deposited, where, first, the large pads, labeled $a-d$, were formed. Then the nanowire, labeled $e$ (length: $100 \mu \mathrm{m}$, width: $80 \mathrm{~nm}$ ) was deposited with a dose of 4.6 $\times 10^{16}$ ions $/ \mathrm{cm}^{2}$ at $20 \mathrm{keV}$ and a probe current of $39 \mathrm{pA}$ to connect pads $a$ and $c$. The beam scanning was controlled by the RAITH software. Under ideal condition, the minimum beam-spot size of the focused-ion beam used in this study has a value of $7 \mathrm{~nm}$. The estimated spot size under the particular condition of a probe current of $39 \mathrm{pA}$ at $20 \mathrm{keV}$ was about $10 \mathrm{~nm}$. Since ion-induced secondary electrons are the primary contributing factor to the chemical vapor deposition process, ${ }^{18}$ for a strip as narrow as $80 \mathrm{~nm}$, we note that the thickness along the wire length direction was largely homogeneous but varied in the width direction. There were no 


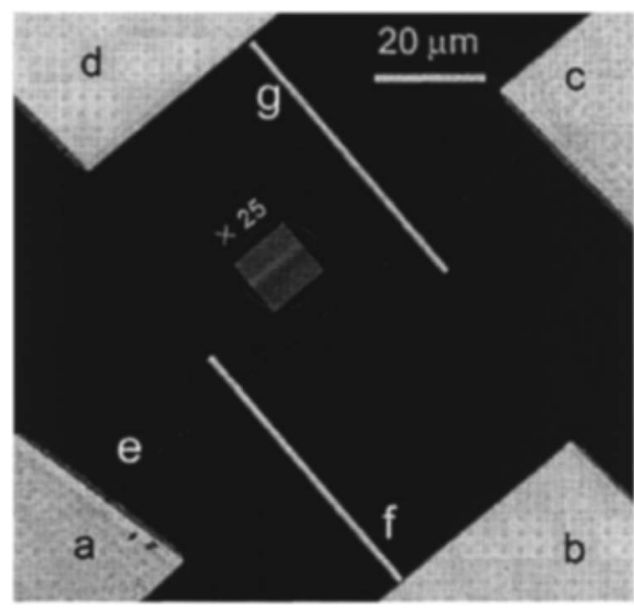

FIG. 1. A typical SEM image of an FIB-induced CVD fabricated W-Ga-C nanowire with $\mathrm{W}-\mathrm{Ga}-\mathrm{C}$ contact electrodes. The four pads are designated as $a, b, c, d$; the nanowire is labeled as $e(80 \mathrm{~nm}$ in width) and the interconnect strips as $f$ and $g$ for simplicity of discussion.

obvious surface roughness, voids, defects, or particles observable at a magnification of $\times 25000$. The strip $f$ was deposited with the same ion source conditions as $e$, but with a wider pattern design, and 60 beam-scanning loops were performed; the relevant dose used was $2.1 \times 10^{19} \mathrm{ions} / \mathrm{cm}^{2}$. The strip $g$ was deposited with the beam-scanning settings exactly the same as those for strip $f$ but a high ion energy was used-an accelerating voltage of $30 \mathrm{keV}$ and a probe current of $80 \mathrm{pA}$-with an estimated dose of $4.3 \times 10^{19} \mathrm{ions} / \mathrm{cm}^{2}$.

Unlike conventional ohmic or Schottky contacts made by photolithography, evaporation, and lift-off techniques, ${ }^{16,19}$ in the present study all contacts and nanowires were fabricated by FIB-induced CVD using the same precursor of metalorganic hexacarbony1 $\left[\mathrm{W}(\mathrm{CO})_{6}\right]$. We found that the deposited material was a mixture of tungsten, carbon, and gallium atoms, in a ratio dependent on the deposition conditions, which is consistent with the results reported recently; ${ }^{13,14,16,17}$ although with our current energydispersive spectroscopy (EDS) it was difficult to determine the exact composition, especially for the smaller structures. In the works by Lin et al. ${ }^{16}$ and $\mathrm{Fu}$ et al.,${ }^{18}$ they suggested that the decomposition and absorption processes during the deposition could cause the attenuation of the intensity of the beam, which in turn would lead to a lesser amount of Ga ions in the substrate and the deposited composite. Therefore, we assume that the incorporation of $\mathrm{Ga}$ in the composite fabricated under low ion energy in this work was not very significant.

The $I-V$ characteristics of the pads and the connecting strips, with a length of $80 \mu \mathrm{m}$, a width of $0.63 \mu \mathrm{m}$, and different ion doses: $5.8 \times 10^{18}$ ions $/ \mathrm{cm}^{2} \quad(\bigcirc) ; 1.2$ $\times 10^{19}$ ions $/ \mathrm{cm}^{2}(\diamond) ; 1.7 \times 10^{19}$ ions $/ \mathrm{cm}^{2}(\nabla)$, as indicated by the different symbols, are shown in Fig. 2(a). These strips show linear $I-V$ behavior, clearly suggesting that contacts to the wires were of ohmic nature. The total resistance measured was $2 \times 10^{4}, 6.6 \times 10^{3}$, and $2.2 \times 10^{3} \Omega$ for the strips deposited with the Ga ion dose of $5.8 \times 10^{18}, 1.2 \times 10^{19}$, and $1.7 \times 10^{19}$ ions $/ \mathrm{cm}^{2}$, respectively. The decrease in resistance with the increase of dose was consistent with the increase in the size of the connecting wires. The nonlinear $I-V$ characteristics $(\triangle)$ at room temperature, shown in the inset of Fig. 2(a), was that of a strip fabricated with a dose of 4.6 Downloaded 11 May 2006 to 146.87.255.19. Redistribution subject
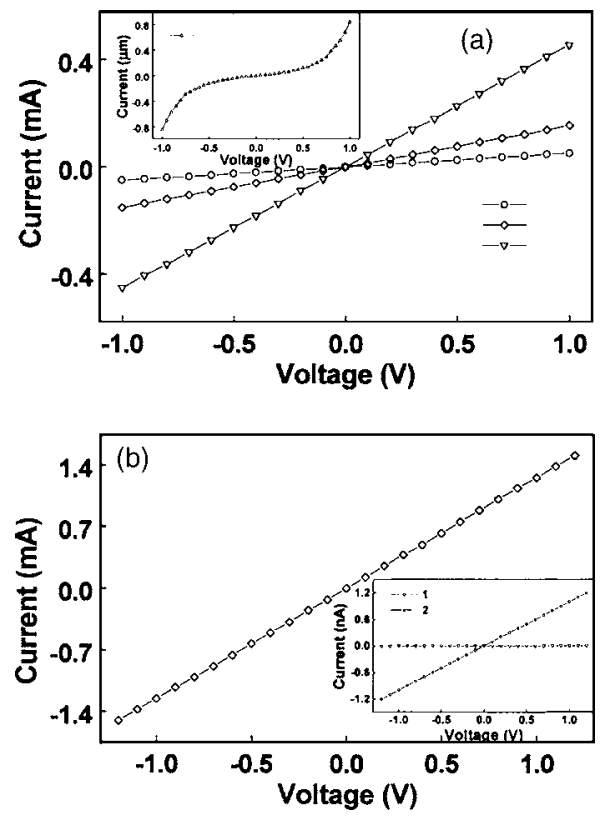

FIG. 2. (a) Room-temperature current-voltage characteristics of contact pads and interconnect strips (length: $80 \mu \mathrm{m}$, width: $0.63 \mu \mathrm{m}$ ) deposited with different ion doses (ions $\left./ \mathrm{cm}^{2}\right)$ : $5.8 \times 10^{18}(\bigcirc) ; 1.2 \times 10^{19}(\diamond) ; 1.7$ $\times 10^{19}(\nabla)$. Inset $(\Delta)$ is the nonlinear $I-V$ curve for the strip with an ion dose of $4.6 \times 10^{16}$ ions $/ \mathrm{cm}^{2}$. (b) Linear $I-V$ curve for a strip (length: $40 \mu \mathrm{m}$, width: $1.25 \mu \mathrm{m}$ ) formed to estimate the resistance of interconnect strip $f$ in Fig. 1. The inset indicates the leakage currents measured by: (curve 1) probes on contact pads without bridge strips and (curve 2) probes placed directly on the substrate.

$\times 10^{16}$ ions $/ \mathrm{cm}^{2}$, suggesting that with further reduction in the wire size different conduction mechanisms might become dominant.

Before we proceed to examine the $I-V$ characteristics of asymmetric contact pairs, we wish to point out that the leakage currents in the substrates were negligible in our study. For instance, the inset in Fig. 2(b) shows the typical leakage currents measured. Curve 1 shows the leakage currents between two pads separated by a distance of $80 \mu \mathrm{m}$ without any bridging connection, which was only about $1.2 \mathrm{nA}$ at $1.2 \mathrm{~V}$. Curve 2 shows the leakage currents when the two probes were placed in direct contact with the $\mathrm{Si}_{3} \mathrm{~N}_{4}$ overlayer, which was of the order of $1 \mathrm{pA}$ for the range of voltages shown in Fig. 2(b). The larger leakage currents between two pads might be due to partial damage to the insulating layer by Ga ions during FIB-induced CVD, which nevertheless were substantially smaller than the currents through a nanowire interconnection. The curve in Fig. 2(b) shows the $I-V$ characteristics of a strip $40 \mu \mathrm{m}$ in length and $1.25 \mu \mathrm{m}$ in width, fabricated using the same conditions as that of the strip $f$ in Fig. 1. From the data, the resistance of the interconnecting strip $f$ was estimated to be of approximately $800 \Omega$, which was negligible compared with the resistance of the nanowire fabricated using a dose of only 4.6 $\times 10^{16}$ ions $/ \mathrm{cm}^{2}$.

In Fig. 3(a), we present the measurements of the $I-V$ characteristics of pairs of contacts along the nanowire, $e$, at room temperature. The inset shows the $I-V$ charateristic (curve 1) between contact pads $a$ and $c$, which were bridged by the long nanowire of $80 \mathrm{~nm}$ in width. Curve 2 illustrates the $I-V$ characteristics of the short nanowire segment with contact electrodes $a$ (positive polarity) and $b$, while curve 3 is that of the long nanowire segment with contact electrodes to AIP license or copyright, see http://apl.aip.org/apl/copyright.jsp 

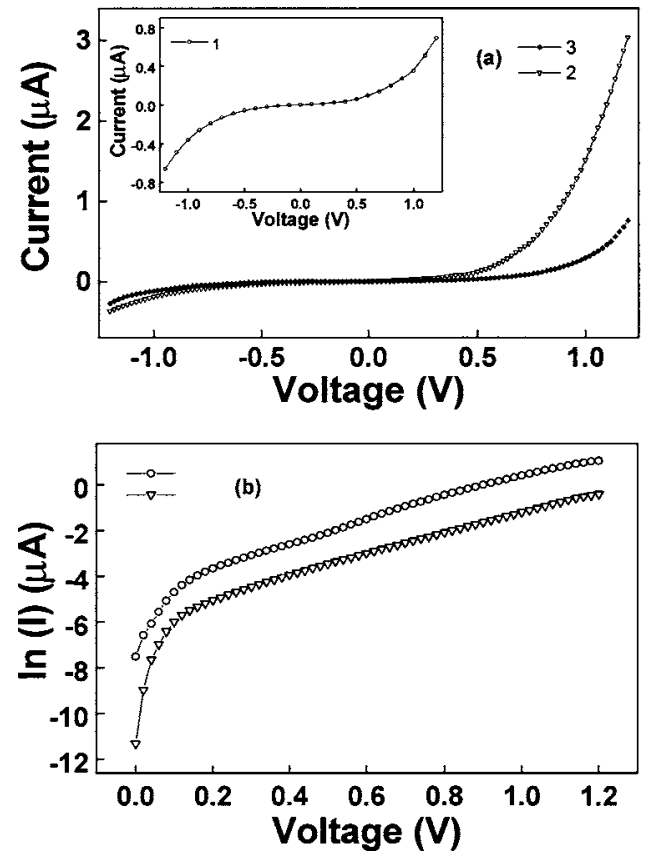

FIG. 3. (a) Room-temperature nonlinear $I-V$ characteristics of (curve 1) the nanowire $e$ with electrodes $a$ and $c$ (positive polarity); (curve 2) short nanowire segment with contact electrodes $a$ (positive) and $b$; (curve 3 ) long nanowire segment with contact electrodes $b$ and $c$ (positive). (b) The natural logarithmic current vs the applied forward bias voltage for contact pairs: $(\bigcirc)$ for $a$ (positive) and $b ;(\nabla)$ for $b$ and $c$ (positive).

$b$ and $c$ (positive). It can be seen from curve 2 in Fig. 3(a) that at $1.2 \mathrm{~V}$, the currents measured at the forward bias voltage were larger by a factor of 8.15 than those measured at the corresponding reverse bias voltage. The result suggested that the contact formed by strip $f$ and the nanowire $e$ was a reasonably good electrical contact, although the contact area was only about $0.1 \mu \mathrm{m}^{2}$. From curves 2 and 3, it could be seen that the resulting characteristics were highly asymmetric and of nonlinear behavior under forward and reverse bias. It could also be seen that at a fixed $V$, the current through the shorter wire segment (between pads $a$ and $b$ ) was much higher than that for the longer wire segment (between pads $c$ and $b$ ) at forward bias of the junctions a/e and $c / e$, showing that the currents were limited by the characteristics of the nanowire. However, at the reverse bias, the difference was much smaller, indicating that the contact barriers at junctions $a / e$ and $c / e$ became dominant. Contact pairs based on interconnecting strip $g$ also showed similar behavior.

Figure 3(b) shows a log-linear plot of the forward bias characteristics at room temperature. For many reasons, the $I-V$ characteristic of a Schottky diode may deviate from the ideal behavior, ${ }^{19}$ which could be manifested by the ideality factor $^{20,21}$ being far from unity. By fitting the data in Fig. 3(b) to the thermionic emission formula, ${ }^{20,21}$ we obtained the ideality factors to be 6.1 and 6.4 , for forward biased contacts $a / e$ and $c / e$, respectively. It is well known that the ideality factor becomes close to unity if thermionic emission is the dominant transport mechanism. In this study, all values of the ideality factor deviated considerably from unity, which indicate that thermionic emission may not be the primary transport mechanism at these junctions. Other carrier transport mechanisms, for instance, tunneling, ${ }^{21}$ may be more important.

In summary, we have fabricated $\mathrm{W}-\mathrm{Ga}-\mathrm{C}$ composite nanohomo contacts by $\mathrm{Ga}$ ion-beam-induced CVD using a dual-beam SEM/FIB. Contact pads to submicron interconnecting strips were found to form good ohmic contacts, whilst those to ultrathin nanowires of about $80 \mathrm{~nm}$ in width showed clear nonlinear $I-V$ characteristics. By introducing the interconnecting strips to the nanowires, asymmetric contact pairs were created showing a clear rectifying behavior. Although the transport mechanisms have yet to be established for such nanohomo contact systems, the results presented here appear to suggest a potential approach for the bottom-up assembly of nanoscale active and passive electronics. Further work is currently underway to explore the possibility and to gain a better understanding of nanocontact formations.

The authors thank Dr. G. Hill at Sheffield University for the deposition of $\mathrm{Si}_{3} \mathrm{~N}_{4}$, Dr. C. Faunce for the EDS measurements, and Yanwei Liu and Paul Cook for various assistance. W.L. acknowledges the Research Scholarship Committee of Salford University for the award of a research studentship. The support of EPSRC and an award from the HEFCE SRIF scheme are also gratefully acknowledged.

${ }^{1}$ X. F. Duan, Y. Huang, Y. Cui, J. F. Wang, and C. M. Lieber, Nature (London) 409, 66 (2001).

${ }^{2}$ J. F. Lin, J. P. Bird, Z. He, P. A. Bennett, and D. J. Smith, Appl. Phys. Lett. 85, 281 (2004).

${ }^{3}$ Y. Huang, X. F. Duan, Y. Cui, L. J. Lauhon, K. H. Kim, and C. M. Lieber, Science 294, 1313 (2001).

${ }^{4}$ R. Martel, T. Schmidt, H. R. Shea, T. Hertel and Rh. Avouris, Appl. Phys. Lett. 73, 2447 (1998).

${ }^{5}$ Z. Yao, H. W. C. Postma, L. Balents, and C. Dekker, Nature (London) 402, 273 (1999).

${ }^{6}$ P. G. Collins, M. S. Arnold and Rh. Avouris, Science 292, 706 (2001).

${ }^{7}$ K. Keem, H. Kim, G. Kim, J. S. Lee, B. Min, K. Cho, M. Y. Sung, and S. Kim, Appl. Phys. Lett. 84, 4376 (2004).

${ }^{8}$ O. Gelhausen, E. Malguth, M. R. Phillips, E. M. Goldys, M. Strassburg, A. Hoffmann, T. Graf, M. Gjukic, and M. Stutzmann, Appl. Phys. Lett. 84, 4541 (2004).

${ }^{9}$ P. Beecher, G. De Marzi, A. J. Quinn, G. Redmond, E. V. Shevchenko, and H. Weller, Appl. Phys. Lett. 85, 5706 (2004).

${ }^{10}$ G. De Marzi, D. Iacopino, A. J. Quinn, and G. Redmond, J. Appl. Phys. 96, 3458 (2004).

${ }^{11}$ Y. Z. Long, Z. J. Chen, W. L. Wang, F. L. Bai, A. Z. Jin, and C. Z. Gu, Appl. Phys. Lett. 86, 153101 (2005).

${ }^{12}$ E. S. Sadki, S. Ooi, and K. Hirata, Appl. Phys. Lett. 85, 6206 (2004).

${ }^{13}$ Z. Q. Liu, K. Mitsuishi, and K. Furuya, J. Appl. Phys. 96, 619 (2004).

${ }^{14}$ Z. Q. Liu, K. Mitsuishi, and K. Furuya, Nanotechnology 15, S414 (2004).

${ }^{15}$ J. F. Lin, J. P. Bird, L. Rotkina, A. Sergeev, and V. Mitin, Appl. Phys. Lett. 84, 3828 (2004).

${ }^{16}$ J. F. Lin, J. P. Bird, L. Rotkina, and P. A. Bennett, Appl. Phys. Lett. 82, 802 (2003).

${ }^{17}$ L. Rotkina, J. F. Lin, and J. P. Bird, Appl. Phys. Lett. 83, 4426 (2003).

${ }^{18}$ Y. Q. Fu and N. K. A. Bryan, J. Vac. Sci. Technol. B 19, 1259 (2001).

${ }^{19}$ S. M. Sze, Semiconductor Devices: Physics and Technology, 2nd ed. (Wiley, New York, 2001), p. 232.

${ }^{20}$ D. Qiao, L. S. Yu, S. S. Lau, J. M. Redwing, J. Y. Lin, and H. X. Jiang, J. Appl. Phys. 87, 801 (2000).

${ }^{21}$ L. S. Yu, Q. J. Xing, D. J. Qiao, S. S. Lau, and J. M. Redwing, J. Appl. Phys. 84, 2099 (1998). 